\title{
ANTI-DYSENTERY ACTIVITY OF TETRACYCLINE IN COMBINATION WITH CURCUMA XANTHORRHIZA ROXB. OR SONCHUS ARVENSIS L
}

\author{
ELIN YULINAH SUKANDAR*, NENG FISHERI KURNIATI, AGUSTINUS BAYU PURNAMA
}

Department of Pharmacology and Clinical Pharmacy, School of Pharmacy, Institute of Technology Bandung, Jawa Barat, Indonesia. Email: elin@fa.itb.ac.id

Received: 11 July 2016, Revised and Accepted: 15 July 2016

\section{ABSTRACT}

Background: Bacterial dysentery, diarrhea caused by Shigella sp. infection, causes a major morbidity and mortality in rural communities of developing countries. In Indonesia, most of the dysentery patients often use antibiotics in combination with herbal medicine to enhance the effect.

Objective: This research aimed to examine the effect of tetracycline in combination with Curcuma xanthorrhiza Roxb. or Sonchus arvensis L. as an anti-dysentery.

Methods: Antibacterial activity of each extract against Shigella flexneri was determined using microdilution method. Furthermore, its activity in combination with tetracycline was determined using checkerboard method. To assess its effect in in vivo, the drug combination was given orally into S. flexneri-infected rat for 7 consecutive days and total plate count in the feces of the rats was determined.

Results: The minimum inhibitory concentration (MIC) of tetracycline, the ethanol extract of $C$. xanthorrhiza and S. arvensis against $S$. flexneri was 4 , 128 , and $512 \mathrm{mg} / 100 \mu \mathrm{L}$, respectively. The combination of each extract with tetracycline could reduce their MIC value, but the total MIC was not lower than 0.5 , suggesting an additive interaction. However, in in vivo study, both combinations could reduce the number of bacteria found in the feces of the rat significantly compared to positive control and to tetracycline only-treated rat at day 6 after administration of the drug, suggesting a synergistic interaction.

Conclusion: A combination of tetracycline with ethanol extract of $C$. xanthorrhiza or with $S$. arvensis is potential to be developed as anti-dysentery drugs against $S$. flexneri.

Keywords: Dysentery, Tetracycline, Curcuma xanthorrhiza, Sonchus arvensis

(C) 2016 The Authors. Published by Innovare Academic Sciences Pvt Ltd. This is an open access article under the CC BY license (http://creativecommons. org/licenses/by/4. 0/) DOI: http://dx.doi.org/10.22159/ajpcr.2016.v9i6.14033

\section{INTRODUCTION}

In developing countries, the incidence of shigellosis is estimated to be 163.2 million cases/year, where 1.1 million deaths occurred. 61\% of all deaths attributable to shigellosis involved children younger than 5 years. The incidence in developing countries is approximately 20 times greater than that in developed countries [1].

Shigella is one of the bacteria that can cause diarrhea, which is known as dysentery. Dysentery is characterized by the presence of blood or mucus and watery stool [2]. All the dysentery episodes should be treated with antibiotic to prevent bacteremia and sepsis [3]. Emergence of multidrug-resistant Shigella spp. (resistance to more than two firstline oral drugs such as ampicillin, co-trimoxazole, and ciprofloxacin) is of growing concern in the world. Therefore, finding an alternative treatment for dysentery is needed to overcome this situation.

Indonesia is rich with its herbal medicines and uses them for disease treatment. Sometimes, Indonesian combines the prescribed medicine with herbal medicine to speed up the treatment processes. In our previous study, we observed that the combination of herbal medicines with certain antibiotics can enhance the antibacterial activity [4]. Therefore in this study, we investigated the anti-dysentery activity of the combination of tetracycline with herbal medicines which are Curcuma xanthorrhiza Roxb. and Sonchus arvensis L.

C. xanthorrhiza and S. arvensis are famous herbal medicines in Indonesia which are used to treat certain diseases. C. xanthorrhiza rhizomes contain terpenoid and curcuminoid compounds [5]. Curcuminoids are beneficial as antioxidant, anti-inflammatory, antibacterial, antitumor, and anticancer [6]. On the other hands, in S. arvensis, polyphenols and sesquiterpene lactones are found to have an antioxidant and an antibacterial activity, respectively $[7,8]$. As both plants have an antibacterial activity, therefore its activity in combination with tetracycline would be investigated further specially its activity as an anti-dysentery.

\section{METHODS}

\section{Materials}

Mueller-Hinton Agar (MHA) (Oxoid Ltd., Hampshire, England) and Mueller-Hinton Broth (MHB) (Oxoid Ltd., Hampshire, England), MacConkey agar (Oxoid Ltd., Hampshire, England) were used as growth media for bacteria. Tetracycline $\mathrm{HCl}$ (as standard antibiotic), ethanol $90 \%, \mathrm{NaCl}$, and carboxymethyl cellulose Na-FSH were purchased from Brataco, Indonesia. Dimethyl sulfoxide (Merck, German) was used for diluting the herbal medicine extract.

\section{Plant collection and identification}

C. xanthorrhiza rhizomes and $S$. arvensis leaves were collected from Cipendey village, Majalengka district and from Banjaran, Bandung district, respectively. Determination of plants was performed in Bandungense Herbarium, School of Life Sciences and Technology, Bandung Institute of Technology. Fresh rhizomes or leaves were sorted, washed, chopped, and dried using oven with temperature $40-45^{\circ} \mathrm{C}$ for several days. Dried rhizomes and leaves then were grounded into a fine powder.

Test microorganism and culture

Shigella flexneri was obtained from the culture collection of School of Pharmacy, Bandung Institute of Technology. S. flexneri was cultured aerobically in MHA and MHB medium. 
Preparation of extract

The crude plant of $C$. xanthorrhiza rhizomes and S. arvensis leaves was extracted by reflux using ethanol $96 \%$ as solvent. The ethanol extract was filtered using Whatman No. 1 and concentrated using rotary evaporator (Buchi R-125) to obtain viscous extract. The viscous extract was then stored at room temperature and protected from sunlight.

\section{Phytochemical screening of ethanolic extract}

The ethanolic extracts were subjected to preliminary phytochemical screening for plant constituents, including examination of alkaloid, flavonoid, quinone, tannin, saponin, and steroid/triterpenoid [9].

\section{Determination the susceptibility of bacteria}

In vitro susceptibility tests were performed in a 96-well microtiter plate to determine minimum inhibitory concentrations (MICs) of the extracts and tetracycline $\mathrm{HCl}$ against $S$. flexneri using standard Broth microdilution methods with a bacteria inoculum of $5 \times 10^{5} \mathrm{CFU} / \mathrm{mL}$ according to the guidelines of Clinical and Laboratory Standards Institute standard M7-A8 [10]. The MIC was defined as the lowest concentration of antimicrobial agent that resulted in the complete inhibition of visible growth. Minimum bactericidal concentrations (MBCs) were established for each test sample. Briefly, medium (approximately $100 \mu \mathrm{L}$ ) from each well showing no visible growth was spread onto MHA plates. Plates were incubated at $37^{\circ} \mathrm{C}$ for $24 \mathrm{hrs}$ or until growth was seen in the growth-positive control plates. MBC was defined as the lowest concentration of antimicrobial agent at which all bacteria in the culture are killed or the lowest concentration of drug that kills $99.9 \%$ of the total initially viable cells [11].

\section{Checkerboard assay}

The antibacterial activity of antibiotics and plant extracts in combination was investigated using the checkerboard broth microdilution method. Two-fold serial dilutions of the antibiotic and two-fold serial dilutions of the plant extracts were prepared for every combination tested and $50 \mu \mathrm{l}$ aliquots of each component was placed into the wells of the sterile 96-well microtiter plate. The inoculum was prepared using the above described MIC determination method. The microtiter plates were then incubated at $37^{\circ} \mathrm{C}$ and MIC was determined after 18-20 hrs of incubation.

The fractional inhibitory concentration (FIC index) for all the combinations was determined using the following formula:

FIC index $=$ FIC $_{\mathrm{A}}+\mathrm{FIC}_{\mathrm{B}}=[\mathrm{A}] / \mathrm{MIC}_{\mathrm{A}}+[\mathrm{B}] / \mathrm{MIC}_{\mathrm{B}}$

FIC $_{A}$ and FIC $_{B}$ are FIC of drug A and B, respectively. While MIC $_{A}$ and MIC $_{B}$ are MIC of drug A and B, respectively. $[\mathrm{A}]$ and $[\mathrm{B}]$ are concentration of drug A and B, respectively. FIC index by checkerboard method is interpreted as follows: $<0.5$ means synergism; $0.5-2$ means additive or indifferent; and $>2$ means antagonism [12].

\section{Animals}

Healthy female Wistar rat, approximately 2 months age and 150-180 g of body weight, were obtained from Bioscience and Biotechnology Research Centre, Bandung Institute of Technology. The animals were fed ad libitum with standard chow and tap water. The study complied the rules of animal handling and experimentation of School of Pharmacy, Bandung Institute of Technology. Throughout the experiments, all animals received human care according to the criteria outlined in the "Institutional Animal Care and Use Committee Guidebook" [13].

\section{Anti-dysentery activity in vivo}

The rats were divided into 4 groups, namely positive control group, tetracycline, combination of tetracycline and C. xanthorrhiza, and the combination of tetracycline and S. arvensis. S. flexneri suspension with $10^{8} \mathrm{CFU} / \mathrm{mL}$ was prepared, and then $1 \mathrm{~mL}$ of suspension was administered orally to each rat. Rats were put into metabolic cage overnight. At next morning, the feces of each rat were collected and then cultured on MacConkey agar to check the growth of S. flexneri. If the induction was successful, the rats were then treated based on their groups for 7 days.

\section{Determination of $S$. flexneri in feces}

After diarrhea induction and drug treatment, all rats were put into the metabolic cage every night and then feces were collected in the morning and weighted. Furthermore, feces were diluted to $10^{6}$ and $10^{7}$. The diluted feces was cultured on MacConkey agar, and then incubated for $18-24 \mathrm{hrs}$ at $35 \pm 2^{\circ} \mathrm{C}$. After $18-24 \mathrm{hrs}$, the number of colony was counted using total plate count method.

\section{Statistical analysis}

Statistical analysis of the results was analyzed with one-way ANOVA followed by least significant difference and Tukey post hoc analysis using SPSS 16.00. $\mathrm{p}<0.05$ were considered to be significant.

\section{RESULTS}

\section{Phytochemical screening of $C$. xanthorrhiza and $S$. arvensis}

Phytochemical screening was done as preliminary steps to determine the presence of chemical compounds in the extract. The results showed that the extract of $C$. xanthorrhiza contained flavonoids, tannins, quinones, saponins, and steroid/triterpenoid. Moreover, the extract of $S$. arvensis contained alkaloid, phenol, flavonoid, tannins, steroids/ triterpenoid, and saponin.

Ethanol extract of $C$. xanthorrhiza has a better antibacterial activity against $S$. flexneri than $S$. arvensis

Antibacterial activity against $S$. flexneri was tested by determining the MIC and MBC of the extract tested. The results showed that C. xanthorrhiza extract had the lowest MIC and MBC value (128 and $2048 \mu \mathrm{g} / \mathrm{mL}$, respectively) than $S$. arvensis extract (Table 1). Tetracycline as a reference showed the best antibacterial activity among both extracts tested, with the MIC value of $4 \mu \mathrm{g} / \mathrm{mL}$ and MBC value of $32 \mu \mathrm{g} / \mathrm{mL}$ (Table 1 ).

C. xanthorrhiza and $S$. arvensis synergize with tetracycline to enhance potency against $S$. flexneri in vitro

Antibacterial activity of the combination of tetracycline with C. xanthorrhiza extract or with $S$. arvensis was further studied using checkerboard method. The results showed that combination of tetracycline and C. xanthorrhiza extract had a reduction of MIC value to $1 / 4$ for tetracycline and $1 / 2$ for $C$. xanthorrhiza. A similar situation was observed in combination of tetracycline with $S$. arvensis (Table 2). Both combinations showed an additive interaction as their FIC index was between 0.5 and 2. To evaluate its activity in vivo, we administered each drug combination into the $S$. flexneri-infected rats.

Combination of tetracycline with $C$. xanthorrhiza extract has less amount of $S$. flexneri found in the rat's feces among tetracycline only and combination of tetracycline with $S$. arvensis treated group Dysentery was induced by injecting $S$. flexneri suspension at day 1 before drug treatment. Next, rats were treated based on their group for 7 consecutive days. The results, which can be seen in Table 3 , showed that positive control group had the highest number of bacteria at day 4 after administration of bacteria suspension, and then it was started to decrease from day 6 and back to normal again at day 8 .

Table 1: MIC and MBC of extracts and tetracycline

\begin{tabular}{lll}
\hline Extract/control & MIC $(\boldsymbol{\mu g} / \mathbf{m L})$ & MBC $(\boldsymbol{\mu g} / \mathbf{m L})$ \\
\hline Ethanol extract of C. xanthorrhiza & 128 & 2048 \\
Ethanol extract of $S$. arvensis & 512 & $>4096$ \\
Tetracycline & 4 & 32 \\
\hline
\end{tabular}

MIC: Minimun inhibitory concentration, MBC: Minimum bactericidal concentration. C. xanthorrhiza: Curcuma xanthorrhiza, S. arvensis: Sonchus arvensis 
Table 2: MIC of $C$. xanthorrhiza and S. arvensis extracts in combination with tetracycline

\begin{tabular}{lllll}
\hline \multirow{2}{*}{ Extracts } & MIC $(\mu \mathrm{g} / \mathbf{m L})$ & \multirow{2}{*}{ FIC } & \\
\cline { 2 - 4 } & Extracts & Tetracycline & \\
\hline Tetracycline - C. xanthorrhiza & $64(1 / 2$ of MIC $)$ & $1(1 / 4$ of MIC $)$ & 0.75 \\
Tetracycline - S. arvensis & $64(1 / 8$ of MIC $)$ & $2(1 / 2$ of MIC $)$ & additive & 0.625 \\
\hline
\end{tabular}

MIC: Minimun inhibitory concentration, FIC: Fractional inhibitory concentration. C. xanthorrhiza: Curcuma xanthorrhiza, S. arvensis: Sonchus arvensis

Table 3: Number of bacteria in feces of $S$. flexneri infected rats

\begin{tabular}{|c|c|c|c|c|c|c|c|c|}
\hline \multirow[t]{2}{*}{ Group } & \multicolumn{8}{|c|}{ Number of bacteria at day } \\
\hline & 1 & 2 & 3 & 4 & 5 & 6 & 7 & 8 \\
\hline Positive control & $15 \pm 14.29$ & $3.7 \pm 2.02$ & $47.3 \pm 37.28$ & $104.2 \pm 167.88$ & $100.2 \pm 44.23$ & $54.7 \pm 59.67$ & $44.5 \pm 43.1$ & $6.5 \pm 12.7$ \\
\hline Tetracycline - C. xanthorrhiza & $10.6 \pm 8.25$ & $23.3 \pm 27.02$ & $15.5 \pm 22.98$ & $14 \pm 20.47$ & $31.8 \pm 52.56$ & $7.8 \pm 7.11$ & $0 \pm 0 *$ & $3.8 \pm 3.35$ \\
\hline Tetracycline - S. arvensis & $1.8 \pm 3.17$ & $15.2 \pm 9.83$ & $4.3 \pm 5.78$ & $138.2 \pm 176.6$ & $44.8 \pm 57.04$ & $11.5 \pm 3.5$ & $4.3 \pm 4.68^{*}$ & $4 \pm 2.53$ \\
\hline
\end{tabular}

Data are presented as average \pm SD, $n=3$ per group, ${ }^{*} \mathrm{p}<0.05$ means significantly different compared to positive control group. S. flexneri: Shigella flexneri,

C. xanthorrhiza: Curcuma xanthorrhiza, S. arvensis: Sonchus arvensis

Tetracycline and combination of tetracycline - S. arvensis group showed the similar pattern as positive control. Combination of tetracycline - C. xanthorrhiza did not show any increase number of bacteria in all observation day. However, the significance different was observed only at day 7 after $S$. flexneri administration. Similar significance response was also observed for combination of tetracycline with $S$. arvensis, although the increase number of bacteria was observed upon bacteria administration. This data suggest that the combination of tetracycline with $C$. xanthorrhiza or with S. arvensis might have potency as an anti-dysentery as it inhibited the increase of number of bacteria significantly at day 7 after $S$. flexneri administration.

\section{DISCUSSION}

In this study, anti-dysentery of combination of tetracycline with C. xanthorrhiza or S. arvensis was determined in vitro and in vivo. The results showed that both combinations had an additive activity against $S$. flexneri in vitro. However, the combination of tetracycline with $C$. xanthorrhiza or S. arvensis showed less number of bacteria found in the rats' feces than those exposed to tetracycline only, suggesting a synergistic interaction in vivo. In conclusion, combination of tetracycline with C. xanthorrhiza or with $S$. arvensis is a potential as anti-dysentery drugs against $S$. flexneri.

Limitation of this study is that there was no pharmacokinetic profile of drugs in combination due to difficulty in finding the plant biomarker to determine the exact concentration of the plant extract in blood stream. Although it is known that to achieve synergism in vivo, sufficient concentration of antibiotics in the bloodstream and infected tissues are important. The concentration of single drug of drug combinations is needed to have a longer half-life to prevent the induction of transient resistance mechanisms and the selection of genetic mutants [14] Therefore, the pharmacokinetic profile of plant extract is needed for further study.

Synergistic interaction of tetracycline with plant extracts has been reported before. Study by Sudano Roccaro, 2004 [15] reported epigallocatechin gallate enhances the tetracycline activity for staphylococci by impairment of tetracycline efflux pump activity and increased intracellular retention of the drug leading to synergistic drug combination. However, the exact mechanism of combination of tetracycline with $C$. xanthorrhiza or S. arvensis against S. flexneri is still under investigation.

\section{CONCLUSION}

The drug combination of tetracycline with C. xanthorrhiza or S. arvensis both shows an additive activity against $S$. flexneri in vitro. However, both combinations have significantly lower number of bacteria found in the rat's feces than those exposed to tetracycline only at day 6 after drug administration, suggesting a synergistic interaction in vivo. Tetracycline combination with each of the ethanol extract of $C$. xanthorrhiza or S. arvensis is potential to be developed as anti-dysentery drugs.

\section{REFERENCES}

1. Sureshbabu J, Venogopalan P, Abuhammour W. Shigella infection, emedicine. Medsape. Available from: http://www.emedicine.medscape. com/article/968773-overview\#a6. [Last accessed on 206 Jun 01].

2. Khalili M. Acute bacterial dysentery in children. Int $\mathrm{J}$ Infect 2014;1:e21589.

3. WHO. Guidelines for the Control of Shigellosis, Including Epidemics Due to Shigella Dysenteriae Type 1. Geneva: World Health Organization; 2005 .

4. Sukandar EY, Kurniati NF, Wikaningtyas P, Agprikani D. Antibacterial interaction of combination of ethanolic extract of Zingiber officinale var. Rubrum rhizome, Boesenbergia pandurata rhizome, and Stevia rebaudiana leaves with certain antibiotics against infectious mouth microbial. Asian J Pharm Clin Res 2016;9:332-5.

5. Angel GR, Vimala B, Nambisan B. Antioxidant and anti-inflammatory activities of proteins isolated from eight Curcuma species. Phytopharmacology 2013;4:96-105.

6. Oon SF, Nallappan M, Tee TT, Shohaimi S, Kassim NK, Sa'ariwijaya MS, et al. Xanthorrhizol: A review of its pharmacological activities and anticancer properties. Cancer Cell Int 2015;15:100.

7. Khan RA. Evaluation of flavonoids and diverse antioxidant activities of Sonchus arvensis. Chem Cent J 2012;6:126.

8. Xia Z, Qu W, Lu H, Fu J, Ren Y, Liang J. Sesquiterpene lactones from Sonchus arvensis L. and their antibacterial activity against Streptococcus mutans ATCC 25175. Fitoterapia 2010;81(15):424-8.

9. Farnsworth NR. Biological and phytochemical screening of plants J Pharm Sci 1966;55(3):225-76.

10. Clinical and Laboratory Standards Intitute. M07-A08 - Methods Dilution Antimicrobial Succeptibility Tests for Bacteria that Grow Aerobically Approve Standard. $8^{\text {th }}$ ed. Pennsylvania: CLSI; 2009.

11. Clinical and Laboratory Standards Intitute. M100-S17 - Performance Standards for Antimicrobial Susceptibility Testing Seventeenth Informational Supplement. Pennsylvania: CLSI; 2007.

12. Pillai SK, Moellering RC, and Eliopoulos GM. Antimicrobial combinations. In: Lorian V, editor. Antibiotics in Laboratory Medicine. $5^{\text {th }}$ ed. Philadelphia: The Lippincott Williams \& Wilkins Co.; 2005. p. $365-440$.

13. Office of Laboratory Animal Welfare, (OLAW). Institutional Animal Care and Use Committee Guidebook. $2^{\text {nd }}$ ed. National Institutes of Health: Applied Research Ethics National Association (ARENA); 2002

14. Bruhn DF, Scherman MS, Liu J, Scherbakov D, Meibohm B, Böttger EC, et al. In vitro and in vivo evaluation of synergism between anti-tubercular spectinamides and non-classical tuberculosis antibiotics. Sci Rep 2015;5:13985.

15. Sudano Roccaro A, Blanco AR, Giuliano F, Rusciano D, Enea V. Epigallocatechin-gallate enhances the activity of tetracycline in staphylococci by inhibiting its efflux from bacterial cells. Antimicrob Agents Chemother 2004;48(6):1968-73 